\title{
No observed effect of homologous recombination on influenza $C$ virus evolution
}

\author{
Guan-Zhu Han ${ }^{1,4^{*}}$, Maciej F Boni ${ }^{2,3}$, Si-Shen $\mathrm{Li}^{1^{*}}$
}

\begin{abstract}
The occurrence of homologous recombination in influenza viruses has been under some debate recently. To determine the extent of homologous recombination in influenza $C$ virus, recombination analyses of all available gene sequences of influenza $C$ virus were carried out. No recombination signal was found. With the previous evidence in influenza $A$ and $B$ viruses, it seems that homologous recombination has minimal or no effect on influenza virus evolution.
\end{abstract}

\section{Background}

The influenza $\mathrm{C}$ virus belongs to the Orthomyxoviridae family and is a common cause of mild upper respiratory tract illness. Seroepidemiological studies indicate that it is widely distributed around the world, but isolated infrequently, and the majority of humans acquire antibodies to the virus early in life [1]. In contrast with influenza $A$ and $B$ viruses, the influenza $C$ virus genome consists of only seven single stranded negative-sense RNA segments, the PB2, PB1, P3, HE, NP, M, and NS segments. Analysis of the full genome sequence of type $C$ influenza viruses suggested that reassortment between two different type $\mathrm{C}$ influenza viruses occurs frequently in nature $[2,3]$. Furthermore, influenza $C$ virus has been suggested to be involved in heterologous RNA recombination events [4].

Largely because their RNA is always encapsidated by a ribonucleoprotein complex (RNP), single-stranded negative-sense RNA viruses are generally believed to undergo a low rate of homologous recombination [5]. However, there is increasing evidence of homologous recombination involving negative-strand RNA viruses like Newcastle disease virus [5-8], Zaire Ebola virus [9], measles virus [10], and canine distemper virus [11,12]. Homologous recombination has also been demonstrated in the laboratory for respiratory syncytial virus and hantavirus $[13,14]$. However, the evidence for homologous recombination in influenza viruses has been sparse and controversial.

\footnotetext{
* Correspondence: guanzhu@email.arizona.edu; ssli@sdau.edu.cn 'State Key Laboratory of Crop Biology, College of Agronomy, Shandong Agricultural University, Tai'an, Shandong 271018, China Full list of author information is available at the end of the article
}

Gibbs et al. proposed that homologous recombination had occurred in the HA gene of 1918 Spanish flu virus [15]. However, the apparent recombination event described by Gibbs et al. is much more likely the result of a difference in the substitution rate between HA1 and HA2 [16]. Several recent studies provide some new evidence for recombination in influenza A virus [17-19]. In particular, He et al. provide evidence for a clade of three recombinant avian influenza sequences [17], but largescale analyses have shown that anomalies in the influenza sequence database, possibly caused by sample contamination, may generate false-positive recombination signals [20-22]. Indeed, when controlling for sequence quality, the evidence for homologous recombination is weak in both influenza A and B viruses [20,22,23]. Given the evidence to date, homologous recombination seems to play little or no role in the evolution of influenza A and B viruses. In a previous small scale analysis, patterns of sequence variation compatible with the action of recombination, but not definitive evidence, were observed in influenza $C$ virus [5]. The increasing availability of genome sequences of influenza $C$ virus may have the potential to shed new light on the role of homologous recombination in the evolution of influenza $C$ virus.

\section{Results and Discussion}

To determine the role of homologous recombination in the evolution of influenza $C$ virus, we gathered all 722 publicly available influenza $C$ virus sequences representing all seven RNA segments on April 10, 2010 (Table 1) and performed recombination analyses as described elsewhere [20]. Briefly, the sequences were obtained from the 
Table 1 Summary of results in this study

\begin{tabular}{llrrr}
\hline Segment & No. of sequences & No. of distinct sequences & Alignment length $(\mathbf{n t})$ & Min Bruen $\boldsymbol{\Phi}$ p-values (window sizes = 50,100,150) \\
\hline PB2 & 90 & 55 & 2365 & 0.43 \\
PB1 & 88 & 49 & 2363 & 0.91 \\
P3 & 88 & 33 & 2183 & 0.24 \\
HE & 138 & 116 & 2075 & 0.74 \\
NP & 87 & 61 & 1809 & 0.86 \\
MP & 108 & 79 & 1180 & 0.50 \\
NS & 123 & 94 & 935 & 0.36 \\
\hline
\end{tabular}

1. All p-values by 3SEQ were one.

2. No. of recombination signals by Chimera, GENECONV and RDP were zero.

Influenza Virus Resource [24] and then aligned using MUSLE v3.6 [25]. All sequence alignments are available from the authors upon request. Recombination signals were tested using the 3SEQ algorithm [26]. 3SEQ tests all possible triplets in a data set for a mosaic recombination signal using a nonparametric statistic for mosaicism, and it reports $\mathrm{p}$-values and breakpoint ranges. 3SEQ's nonparametric $\Delta$-statistic is a special type of Mann-Whitney U-test on binary outcomes where, rather than identifying one outcome coming sequentially before another outcome, one outcome is identified to cluster in the middle of the sequence of the other outcomes. For example, both the Mann-Whitney U-test and 3SEQ's $\Delta$-statistic would identify 000010000111101111 as a statistically significant pattern where the $1 \mathrm{~s}$ appear sequentially after the 0s; however, only the $\Delta$-statistic would identify 000111011101100000 as a statistically significant sequence where the $1 \mathrm{~s}$ cluster in the middle of a sequence of $0 \mathrm{~s}$. This latter sequence of zeros and ones is the type of pattern that must be detected with recombination software that identifies mosaic signals.

3SEQ is among the most powerful recombination detection methods, especially in datasets with high nucleotide diversity [26]. Mean pairwise distances for the influenza $C$ data sets analyzed here ranged from $7 \mathrm{nt}$ to $75 \mathrm{nt}$, and it is possible that for this range of diversity 3SEQ would not have enough power to detect recombination. To determine if recombination could not be found because of the low diversity of influenza $C$ viruses, we tested all seven segments with a homoplasy method (Bruen's $\Phi$ [27]) as homoplasy methods are intended to detect recombination in data sets with low levels of diversity. Window sizes for this methods were set to 50,100 , and 150 , and no recombination signal was found, as all p-values were greater than 0.24 (Table 1). In addition, recombination detection was also performed by using Chimaera, GENECONV, and RDP, which are available in the RDP (Recombination Detection Program) software package [28]. None of the seven influenza $C$ virus data sets analyzed here contained sequences with statistically significant recombination signals (Table 1).
In this study, we used all influenza $C$ viral sequences available in Influenza Virus Resource. Although the majority of segments PB1, PB2, P3, and NP sequences are incomplete, their lengths are enough to find signals if recombination did occur frequently [27]; if recombination occurred infrequently, then the low levels of nucleotide diversity for some influenza $\mathrm{C}$ segments may make it difficult to detect recombination signals.

Influenza viruses evolve through a variety of genetic mechanisms, rapid mutation, frequent reassortment and rarely non-homologous recombination [29]. Homologous recombination has been proposed as an important evolutionary force for evolution of influenza A virus $[17,18]$, but the evidence to date has been weak and few studies have carried out quality-controlled experiments to determine whether homologous recombination is present or extensive in influenza virus evolution [22]. Here we demonstrate that, given the limited sequence diversity in current sequence data, there are no observed homologous recombination signals for influenza $C$ viruses.

\section{Conclusion}

In our study, no homologous recombination signal was found in influenza $C$ virus. Given the present evidence, homologous recombination, if it exists, may only play a minor role in the evolution of influenza virus.

\section{Acknowledgements}

We thank Ms. Xiping Liu for her help during performing this study.

\section{Author details}

${ }^{1}$ State Key Laboratory of Crop Biology, College of Agronomy, Shandong Agricultural University, Tai'an, Shandong 271018, China. ${ }^{2}$ Oxford University Clinical Research Unit, Ho Chi Minh City, Vietnam. ${ }^{3} \mathrm{MRC}$ Centre for Genomics and Global Health, University of Oxford, Oxford, UK. ${ }^{4}$ Current Address: Department of Ecology and Evolutionary Biology, University of Arizona, Tucson, Arizona 85721, USA.

\section{Authors' contributions}

GZH, SSL designed the study; GZH, MFB performed the research; GZH drafted the manuscript. MFB, SSL revised the manuscript. All authors read and approved the final manuscript.

\section{Competing interests}

The authors declare that they have no competing interests. 
Received: 28 May 2010 Accepted: 14 September 2010

Published: 14 September 2010

\section{References}

1. Matsuzaki Y, Katsushima N, Nagai Y, Shoji M, Itagaki T, Sakamoto M, Kitaoka S, Mizuta K, Nishimura H: Clinical features of influenza C virus infection in children. J Infect Dis 2006, 193:1229-1235.

2. Peng $G$, Hongo S, Kimura H, Muraki Y, Sugawara K, Kitame F, Numazaki $Y$, Suzuki $\mathrm{H}$, Nakamura K: Frequent occurrence of genetic reassortment between influenza C virus strains in nature. J Gen Virol 1996, 77:1489-1492.

3. Matsuzaki $Y$, Mizuta $K$, Sugawara $K$, Tsuchiya E, Muraki $Y$, Hongo S, Suzuki $H$, Nishimura H: Frequent reassortment among influenza C viruses. J Virol 2003, 77:871-881.

4. Luytjes W, Bredenbeek PJ, Noten AF, Horzinek MC, Spaan WJ: Sequence of mouse hepatitis virus A59 mRNA 2: indications for RNA recombination between coronaviruses and influenza C virus. Virology 1988, 166:415-422.

5. Chare ER, Gould EA, Holmes EC: Phylogenetic analysis reveals a low rate of homologous recombination in negative-sense RNA viruses. J Gen Virol 2003, 84:2691-2703.

6. Qin Z, Sun L, Ma B, Cui Z, Zhu Y, Kitamura Y, Liu W: F gene recombination between genotype II and VII Newcastle disease virus. Virus Res 2008, 131:299-303.

7. Han G, He C, Ding N, Ma L: Identification of a natural multi-recombinant of Newcastle disease virus. Virology 2008, 371:54-60.

8. Zhang R, Wang X, Su J, Zhao J, Zhang G: Isolation and analysis of two naturally-occurring multi-recombination Newcastle disease viruses in China. Virus Res 2010, 151:45-53.

9. Wittmann TJ, Biek R, Hassanin A, Rouquet P, Reed P, Yaba P, Pourrut X, Real LA, Gonzalez JP, Leroy EM: Isolates of Zaire ebolavirus from wild apes reveal genetic lineage and recombinants. Proc Natl Acad Sci USA 2007, 104:17123-17127.

10. Schierup MH, Mordhorst CH, Muller CP, Christensen LS: Evidence of recombination among early-vaccination era measles virus strains. $B M C$ Evol Biol 2005, 5:52.

11. McCarthy AJ, Shaw MA, Goodman SJ: Pathogen evolution and disease emergence in carnivores. Proc Biol Sci 2007, 274:3165-3174.

12. Han G, Liu X, Li S: Cross-species recombination in the haemagglutinin gene of canine distemper virus. Virus Res 2008, 136:198-201.

13. Spann KM, Collins PL, Teng MN: Genetic recombination during coinfection of two mutants of human respiratory syncytial virus. J Virol 2003, 77:11201-11211.

14. Plyusnin A, Kukkonen SKJ, Plyusnina A, Vapalahti O, Vaheri A: TransfectionMediated Generation of Functionally Competent Tula Hantavirus with Recombinant S RNA Segment. EMBO J 2002, 21:1497-1503.

15. Gibbs MJ, Armstrong JS, Gibbs AJ: Recombination in the hemagglutinin gene of the 1918 "Spanish flu". Science 2001, 293:1842-1845.

16. Worobey M, Rambaut A, Pybus OG, Robertson DL: Questioning the evidence for genetic recombination in the 1918 "Spanish flu" virus. Science 2002, 296:211.

17. He C, Xie Z, Han G, Dong J, Wang D, Liu J, Ma L, Tang X, Liu X, Pang Y, $\mathrm{Li} \mathrm{G}$ : Homologous recombination as an evolutionary force in the avian influenza A virus. Mol Biol Evol 2009, 26:177-187.

18. He C, Han G, Wang D, Liu W, Li G, Liu X, Ding N: Homologous Recombination Evidence in Human and Swine Influenza A Viruses. Virology 2008, 380:12-20.

19. Liu X, Wu C, Chen A: Codon usage bias and recombination events for neuraminidase and hemagglutinin genes in Chinese isolates of influenza A virus subtype H9N2. Arch Virol .

20. Boni MF, Zhou Y, Taubenberger JK, Holmes EC: Homologous Recombination is Very Rare or Absent in Human Influenza A Virus. J Virol 2008, 82:4807-4811.

21. Krasnitz M, Levine AJ, Rabadan R: Anomalies in the Influenza Virus Genome Database: New Biology or Laboratory Errors? I Virol 2008, 82:8947-8950.

22. Boni MF, de Jong MD, van Doorn HR, Holmes EC: Guidelines for Identifying Homologous Recombination Events in Influenza A Virus. PLoS ONE 2010, 5:e10434.

23. Han G, Liu X, Li S: Homologous recombination is unlikely to play a major role in influenza B virus evolution. Virology J 2008, 5:65.
24. The Influenza Virus Resource. [http://www.ncbi.n/m.nih.gov/genomes/FLU/ Database/select.cgi].

25. Edgar RC: MUSCLE: multiple sequence alignent with high accuracy and high throughput. Nucleic Acids Res 2004, 32:1792-1797.

26. Boni MF, Posada D, Feldman MW: An exact nonparametric method for inferring mosaic structure in sequence triplets. Genetics 2007, 176:1035-1047.

27. Bruen TC, Philippe H, Bryant D: A simple and robust statistical test for detecting the presence of recombination. Genetics 2006, 172:2665-2681.

28. Martin DP, Williamson C, Posada D: RDP2: recombination detection and analysis from sequence alignments. Bioinformatics 2005, 21:260-262.

29. Nelson Ml, Holmes EC: The evolution of epidemic influenza. Nat Rev Genet 2007, 8:196-205.

doi:10.1186/1743-422X-7-227

Cite this article as: Han et al:: No observed effect of homologous recombination on influenza C virus evolution. Virology Journal 2010 7:227.

\section{Submit your next manuscript to BioMed Central and take full advantage of:}

- Convenient online submission

- Thorough peer review

- No space constraints or color figure charges

- Immediate publication on acceptance

- Inclusion in PubMed, CAS, Scopus and Google Scholar

- Research which is freely available for redistribution 